\title{
LONG WAVELENGTH VORTICES IN TIME-PERIODIC FLOWS
}

\author{
ANDREW P. BASSOM ${ }^{1}$ and P. J. BLENNERHASSETT ${ }^{2}$
}

(Received 18 September 1996; revised 18 February 1997)

\begin{abstract}
The linear stability properties are examined of long wavelength vortex modes in two timeperiodic flows. These flows are the motion which is induced by a torsionally oscillating cylinder within a viscous fluid and, second, the flow which results from the sinusoidal heating of an infinite layer of fluid. Previous studies concerning these particular configurations have shown that they are susceptible to vortex motions and linear neutral curves have been computed for wavenumbers near their critical value. These computations become increasingly difficult for long wavelength motions and here we consider such modes using asymptotic methods. These yield simple results which are formally valid for small wavenumbers and we show that the agreement between these asymptotes and numerical solutions is good for surprisingly large wavenumbers. The two problems studied share a number of common features but also have important differences and, between them, our methods and results provide a basis which can be extended for use with other time-periodic flows.
\end{abstract}

\section{Introduction}

Temporally periodic flows occur in numerous important contexts and have applications in a diverse range of fields including engineering, geophysical dynamics and physiology. Two relatively simple examples, on which we shall concentrate here, are the Stokes layer which is induced by an infinitely long, torsionally oscillating cylinder within an otherwise quiescent viscous fluid of infinite extent, and a thermal analogue which arises from the oscillatory heating of a horizontal boundary of a semi-infinite region of fluid. In the case of the oscillating cylinder, fluid motion is confined to a thin layer adjacent to the cylinder with the velocity field being exponentially small at large distances form the cylinder. Similarly, the temperature field far from the heated boundary is uniform, with significant temperature variation being confined to a thin region close to the boundary. The stability of practically relevant flows must

\footnotetext{
'Department of Mathematics, University of Exeter, North Park Road, Exeter, Devon, EX4 4QE, UK

${ }^{2}$ School of Mathematics, University of New South Wales, Sydney, NSW 2052, Australia

(C) Australian Mathematical Society, 1998, Serial-fee code 0334-2700/98
} 
always be a topic of concern and the objective of the current study is to examine the stability properties of temporally periodic flows when subjected to small amplitude, long wavelength vortex-like disturbances.

The curved Stokes layer and the sinusoidally heated semi-infinite layer are known to be susceptible to vortex instabilities. (For the sake of brevity we shall hereafter refer to these problems as the 'cylinder' and 'plane layer' problems respectively.) In the former case the instability appears as a structure which is periodic in the direction of the cylinder axis and which is confined to a relatively thin zone on the surface of the cylinder. For the plane layer problem the instability is periodic across the plate and varies in the direction normal to the bounding surface. Computations by Seminara and Hall [4] and Hall [2] have yielded linear neutral stability curves for the respective cases. Under the periodicity constraints mentioned above, it is a straightforward task to show that the requisite stability equations take the forms of partial differential systems involving one space variable and time (details of these systems are given below). Solutions were obtained by writing the dependent variables as Fourier series in time with coefficients functions of the space co-ordinate. By substituting these expressions into the governing system, and then comparing the coefficients of the individual harmonics, each problem can be re-cast as an infinity of coupled ordinary differential equations for the coefficients in the assumed Fourier series solution.

Seminara and Hall [4] solved the curved Stokes layer problem by approximating the solution by finite Fourier-like series and thereby reducing the formally infinite family of ordinary differential equations to a finite set. These were then tackled using shooting methods coupled to Newton iteration. For vortex wavenumbers around critical this procedure works well but it becomes increasingly sensitive and inconvenient to use as the wavenumber becomes large. The principal reason for this is that at this wavenumber extreme the level at which truncation should be applied in order to produce reliable results rapidly becomes excessive. Then one is left with a very large system of equations and, even with modern computing resources, accurate solutions are not easily obtained.

A recent study by Blennerhassett et al. [1] has shown that in the high wavenumber (or, equivalently, the short wavelength) limit the impractical Fourier decomposition method of solution is superseded by a WKB-like approach to the analysis of the -problems. This technique, although formally only justifiable for asymptotically short wavelengths, turns out to yield useful information about the neutral stability curve for a surprisingly large band of wavenumbers. Even for the most straightforward time-periodic flows this WKB approach is technically involved in detail but, having appreciated its general properties, the application of its results to practically relevant flows is not difficult. This was illustrated in [1] where, although the general theory was developed by appealing to a simplified model, it was shown that it may easily be applied to the oscillating cylinder and heated plane layer problems. The purpose of 
the present study is to complete the investigation of the linear vortex stability of time periodic flows by turning attention to the small wavenumber limit. It is found that this is somewhat simpler to analyse than the corresponding large wavenumber case but, nonetheless, it contains a number of interesting features. As in [1], we shall illustrate our methods by applying them to the two particular instances already described and, whilst the basic solution philosophy is the same in each case, the respective solutions have a number of contrasting features. Given this additional analysis, it is then theoretically possible to examine the vortex stability of a large class of temporally periodic flows across the entire wavenumber spectrum. For $O(1)$ wavenumbers a numerical solution of the Fourier decomposed equations is necessary whilst for large or small wavelengths the asymptotic arguments given herein or by [1] are required.

The remainder of the paper is organised as follows. In Section 2 we outline the formulation of the linear stability equations for the curved Stokes flow, discuss their numerical solution and analyse the long wavelength limit. A similar pattern is taken in Section 3 for the sinusoidally heated infinite layer of fluid and we conclude in Section 4 with some discussion and comparison of the two problems.

\section{The curved Stokes flow}

Consider the flow which is induced by a torsionally oscillating cylinder of radius $R$ and infinite length in an incompressible fluid of density $\rho$ and kinematic viscosity $\nu$. Relative to the usual cylindrical polar co-ordinates $(r, \theta, z)$ aligned so that the $z$-axis coincides with the axis of the cylinder, it is well known that for high frequencies of oscillation $\omega$ the induced fluid motion is confined to the region where $r-R$ is $O(\sqrt{\nu / \omega})$. Accordingly, it is convenient to define dimensionless co-ordinates $\eta$ and $Z$ by

$$
\eta=\sqrt{\frac{\omega}{2 v}}(r-R), \quad z=\sqrt{\frac{\omega}{2 v}} Z
$$

and suppose that the fluid velocities in the $r-, \theta-$ and $z-$ directions are written

$$
\boldsymbol{u}=(\sqrt{\nu \omega} u, \Delta \omega v, \sqrt{\nu \omega} w)
$$

Here $\Delta$ denotes the amplitude of the oscillation of the cylinder and, in the limit when $R^{-1} \sqrt{\nu / \omega}$ is small, the relative importance of the centripetal forces tends to vanish, so that at the lowest order of approximation the basic flow is the classical Stokes layer

$$
u=w=0, \quad v=v_{B}=\exp (-\eta) \cos (t-\eta),
$$

where $t$ is a dimensionless time based on the oscillation frequency. If this flow is disturbed by infinitesimal amplitude vortices of axial wavenumber $a$ then the velocity 
components may be written

$$
\begin{gathered}
(u, v, w)=\left(0, v_{B}, 0\right) \\
+\delta[(\hat{u}(\eta, t), \hat{v}(\eta, t), \hat{w}(\eta, t)) \exp (\mathrm{i} a z)+\text { complex conjugate }], \\
\delta \ll 1 .
\end{gathered}
$$

By substituting (2.4) into the continuity and Navier-Stokes equations and linearising, one derives the usual disturbance equations

$$
\begin{array}{r}
\left(\frac{\partial^{2}}{\partial \eta^{2}}-a^{2}-2 \frac{\partial}{\partial t}\right)\left(\frac{\partial^{2}}{\partial \eta^{2}}-a^{2}\right) \hat{u}-2 a^{2} T v_{B} \hat{v}=0 \\
\left(\frac{\partial^{2}}{\partial \eta^{2}}-a^{2}-2 \frac{\partial}{\partial t}\right) \hat{v}-\sqrt{2} \frac{\partial v_{B}}{\partial \eta} \hat{u}=0
\end{array}
$$

which need to be solved subject to $\hat{u}=\hat{v}=\partial \hat{u} / \partial \eta=0$ at $\eta=0$ and as $\eta \rightarrow \infty$. In these equations $T$ is the Taylor number based on the Stokes layer thickness and is defined by $T \equiv 2 \Delta^{2} \sqrt{\omega} /(R \sqrt{v})$.

Equations (2.5) with the associated boundary conditions are precisely the forms which were solved numerically by Seminara and Hall [4]. For O(1) values of the wavenumber, the natural way to isolate neutral modes is to decompose $\hat{u}$ and $\hat{v}$ into Fourier time series and thereby reduce (2.5) to an infinite set of coupled ordinary differential equations. If we write

$$
(\hat{u}, \hat{v})=\sum_{n=-\infty}^{\infty}\left(U_{n}(\eta), V_{n}(\eta)\right) e^{\mathrm{i} n t}
$$

the governing equations become

$$
\begin{gathered}
\left(D^{2}-a^{2}-2 \mathrm{i} n\right)\left(D^{2}-a^{2}\right) U_{n}-a^{2} T\left[e^{-\eta(1+\mathrm{i})} V_{n-1}+e^{-\eta(1-\mathrm{i})} V_{n+1}\right]=0 \\
\left(D^{2}-a^{2}-2 \mathrm{i} n\right) V_{n}+\frac{1}{\sqrt{2}}\left[(1+\mathrm{i}) e^{-\eta(1+\mathrm{i})} U_{n-1}+(1-\mathrm{i}) e^{-\eta(1-\mathrm{i})} U_{n+1}\right]=0 \\
U_{n}=D U_{n}=V_{n}=0 \quad \text { at } \eta=0 \quad \text { and } \quad \text { as } \eta \rightarrow \infty
\end{gathered}
$$

where $D$ denotes $d / d \eta$. Seminara and Hall [4] derived formal infinite double-series solutions for each harmonic $U_{n}$ and $V_{n}$ which show that the solution components decay exponentially as $\eta \rightarrow \infty$.

The system (2.6) is solved by choosing $U_{n}=V_{n} \equiv 0$ for all $|n| \geq N$ at some level of truncation $N$ and the remaining finite-dimensional system treated as an eigenproblem for $T=T(a)$. Seminara and Hall [4] found that the lowest value of $T$ occurred at 
the critical wavenumber $a_{c} \approx 0.8585$ with corresponding Taylor number $T_{c} \approx 164.41$ and that $T \rightarrow \infty$ as $a \rightarrow 0$ or $\infty$. Severe numerical problems were encountered at wavenumbers greater than about $2 a_{c}$ for then the level of truncation demanded by system (2.6) in order to achieve reliable results is so great as to make the computation infeasible.

Numerical difficulties also arise as the wavenumber becomes much smaller than $a_{c}$. An examination of the governing system shows that the $U_{n}$ velocity components decay like $e^{-a \eta}$ for large $\eta$ and so, for $a$ small, the size of the computational domain must be increased to accommodate the slowly decaying components of the solution. This computational difficulty can be overcome by the use of projection conditions, as described by Lentini and Keller [3], at the far-field boundary, rather than directly imposing the conditions as stated in (2.6c). These projection conditions allow a much smaller computational domain to be used, but its size is still weakly dependent on the wavenumber $a$. The results presented in Figures 2 and 4 below and described as the solutions of the full eigenvalue problems were obtained using the above techniques, as well as the inefficient method of simply using $(0,10 / a)$ as the computational domain. The main drawback of the numerical solution is that it completely ignores the structure of the perturbations as the wavenumber $a$ tends to zero. In this limit the solution develops a two-layer structure, where the appropriate $O(1)$ co-ordinates are $\eta$, for the layer on the cylinder, and $Y=a \eta$ for the far field. The solution in the far field can be obtained analytically (as the Stokes layer velocity has decayed to zero), and matching this to the wall layer provides boundary conditions for a computational problem for which the size of the $\eta$ domain is no longer dependent on the wavenumber. The details of this process are given below.

For the small $a$ limit we begin by expanding each variable $U_{n}, V_{n}$ as power series in $a$. Following Seminara and Hall [4], we exploit the fact that (2.6) can be decoupled into two systems; one involving even indexed $U$ components and odd indexed $V$ terms and the other vice-versa. It is well established that the more dangerous system (in the sense that for given wavenumber it becomes unstable at the lower Taylor number) is the first of these so we set $U_{2 r+1}=V_{2 r}=0$ for all integers $r$. (In addition, the harmonics of the solution satisfy $U_{n}=U_{-n}^{*}, V_{n}=V_{-n}^{*}$ where the asterisk denotes complex conjugation.) The unknowns are now expanded as

$$
U_{2 n}=u_{2 n}+a \phi_{2 n}+\ldots, \quad V_{2 n+1}=v_{2 n+1}+a \psi_{2 n+1}+\ldots,
$$

with

$$
T=T_{0} a^{-2}+T_{1} a^{-1}+\ldots,
$$


so that the governing equations for the leading order terms become

$$
\begin{aligned}
\left(D^{2}-4 \mathrm{i} n\right) D^{2} u_{2 n}-T_{0}\left[e^{-\eta(1+\mathrm{i})} v_{2 n-1}+e^{-\eta(1-\mathrm{i})} v_{2 n+1}\right] & =0 \\
\left(D^{2}-2 \mathrm{i}(2 n+1)\right) v_{2 n+1}+\left[(1+\mathrm{i}) e^{-\eta(1+\mathrm{i})} u_{2 n}+(1-\mathrm{i}) e^{-\eta(1-\mathrm{i})} u_{2 n+2}\right] / \sqrt{2} & =0
\end{aligned}
$$

with the $O(a)$ correction terms governed by

$$
\begin{gathered}
\left(D^{2}-4 \mathrm{i} n\right) D^{2} \phi_{2 n}-T_{0}\left[e^{-\eta(1+\mathrm{i})} \psi_{2 n-1}+e^{-\eta(1-\mathrm{i})} \psi_{2 n+1}\right] \\
=T_{1}\left[e^{-\eta(1+\mathrm{i})} v_{2 n-1}+e^{-\eta(1-\mathrm{i})} v_{2 n+1}\right] \\
\left(D^{2}-2 \mathrm{i}(2 n+1)\right) \psi_{2 n+1}+\left[(1+\mathrm{i}) e^{-\eta(1+\mathrm{i})} \phi_{2 n}+(1-\mathrm{i}) e^{-\eta(1-\mathrm{i})} \phi_{2 n+2}\right] / \sqrt{2}=0
\end{gathered}
$$

The leading order equations $(2.8 \mathrm{a}, \mathrm{b})$ need to be solved subject to the boundary conditions

$$
\begin{gathered}
u_{2 n}=D u_{2 n}=v_{2 n+1}=0 \quad \text { at } \eta=0, \\
u_{0}-C_{0} \eta \rightarrow \lambda_{0}, \quad u_{2 n} \rightarrow C_{2 n}, \quad v_{2 n+1} \rightarrow 0 \quad \text { as } \eta \rightarrow \infty
\end{gathered}
$$

for some constants $\lambda_{0}, C_{2 n}$. We note that the conditions $(2.9 \mathrm{~b})$ do not force the disturbance velocities to vanish as $\eta \rightarrow \infty$, and yet neither do they allow the most general behaviour of $u_{2 n}$ consistent with the governing equations. Clearly equation (2.8a) allows $u_{2 n}$ to depend linearly on $\eta$ for all $n$, but it was found that only solutions satisfying (2.9b) could be matched to solutions in the outer region where $Y=a \eta$ is $O(1)$. Thus, in this outer region, the solution takes the form

$$
U_{0}=a^{-1} \hat{u}_{0}(Y)+\ldots \text { and } U_{2 n}=\hat{u}_{2 n}(Y)+\ldots \text { for } n \neq 0,
$$

with all the $V_{2 n+1}$ components being exponentially small; in fact they are of size $\exp \left(-O\left(a^{-1}\right)\right)$. The substitution of $(2.10)$ into the governing equations $(2.6 \mathrm{a}, \mathrm{b})$ and the matching of the resulting outer solutions to the inner forms as $Y \rightarrow 0$ gives the results

$$
\hat{u}_{0}=C_{0} Y e^{-Y}, \quad \hat{u}_{2 n}=C_{2 n} e^{-Y} \quad(n \neq 0) .
$$

It is now clear that this outer zone ensures that the solutions in the region where $\eta \sim O(1)$ are brought smoothly to zero in the far field.

Equations $(2.8 \mathrm{a}, \mathrm{b})$, subject to $(2.9 \mathrm{a}, \mathrm{b})$, were solved on the finite domain $\left(0, \eta_{\infty}\right)$ using the boundary value routines of the AUTO package. As $\eta \rightarrow \infty$ the governing equations decouple completely, and the boundary conditions at infinity $(2.9 \mathrm{~b})$ could be implemented as conditions prohibiting exponential growth of the solutions at $\eta_{\infty}$. The usual checks were made that the numerical solution had converged adequately 


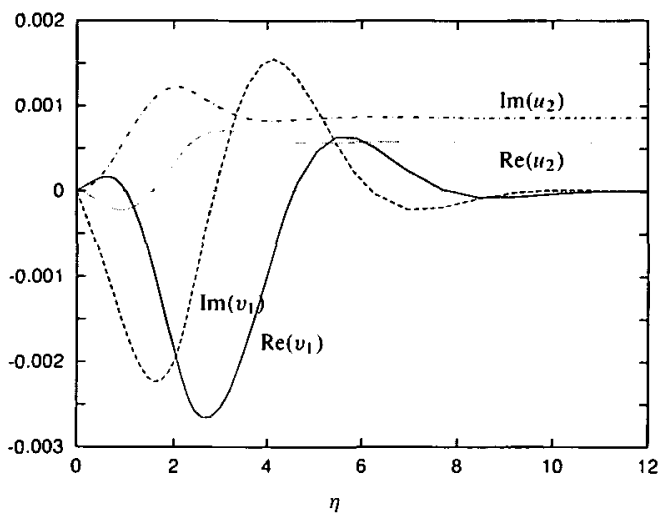

FIGURE 1. The eigenfunctions $v_{1}(\eta)$ and $u_{2}(\eta)$ of the leading order eigenproblem $(2.8 \mathrm{a}, \mathrm{b}),(2.9)$. The real and imaginary parts of $v_{1}$ are denoted by the solid and dashed lines respectively whilst the corresponding components of $u_{2}$ are shown by the dotted and dot-dash lines.

and that the level of truncation of both the Fourier series and the flow domain was sufficiently high that the predicted value of $T_{0}$ was independent of the truncation points. In practice taking components up to $v_{7}$ was more than sufficient and a value of $\eta_{\infty}=12$ worked well. The value of $T_{0}$ was found to be

$$
T_{0} \approx 12.284
$$

and Figure 1 shows the real and imaginary parts of the harmonics $v_{1}$ and $u_{2}$. We note that $u_{2}$ has attained its asymptotic value well before $\eta_{\infty}$, and that $v_{1}$ has just decayed to zero at the end of the computational domain. This figure represents the worst case for the decay of the $v_{2 n+1}$ components, as higher harmonics in $v$ decay with increasing speed, as can be seen by examining the governing equations at large $\eta$. Similarly, higher harmonics in $u$ reach their large $\eta$ asymptote faster than $u_{2}$. By way of comparison, the constant $C_{0}$ in $(2.9 \mathrm{~b})$ is approximately 0.046 , so that at $\eta_{\infty}$ the mean $u$ velocity is some 400 times larger than $u_{2}$ so, in fact, $u_{0}$ dominates the solution.

In order to derive an expression for the correction term $T_{1}$ in the Taylor number expansion (2.7c) the system (2.8c,d) for the functions $\phi_{2 n}, \psi_{2 n+1}$ is considered. The conditions which must be met on the surface of the cylinder $\eta=0$ and those enforced by matching with the outer solution necessitate that

$$
\begin{gathered}
\phi_{2 n}=D \phi_{2 n}=\psi_{2 n+1}=0 \quad \text { on } \quad \eta=0 \\
D^{2} \phi_{0} \rightarrow-2 C_{0}, \quad D \phi_{2 n} \rightarrow-C_{2 n}, \quad \psi_{2 n+1} \rightarrow 0 \quad \text { as } \quad \eta \rightarrow \infty
\end{gathered}
$$


The value of $T_{1}$ is determined by the standard procedure of examination of adjoint functions. The system which is adjoint to $(2.8 \mathrm{a}, \mathrm{b}),(2.9 \mathrm{a}, \mathrm{b})$ is given by

$$
\begin{array}{r}
\left(D^{2}-4 \mathrm{i} n\right) D^{2} \alpha_{2 n}+\left[(1+\mathrm{i}) e^{-\eta(1+\mathrm{i})} \beta_{2 n+1}+(1-\mathrm{i}) e^{-\eta(1-\mathrm{i})} \beta_{2 n-1}\right] / \sqrt{2}=0 \\
\left(D^{2}-2 \mathrm{i}(2 n+1)\right) \beta_{2 n+1}-T_{0}\left[e^{-\eta(1+\mathrm{i})} \alpha_{2 n+2}+e^{-\eta(1-\mathrm{i})} \alpha_{2 n}\right]=0
\end{array}
$$

subject to

$$
\begin{gathered}
\alpha_{2 n}=D \alpha_{2 n}=\beta_{2 n+1}=0 \quad \text { at } \quad \eta=0, \\
D \alpha_{0} \rightarrow \bar{\alpha}_{0}^{\prime}, \quad \alpha_{2 n} \rightarrow \bar{\alpha}_{2 n}, \quad \beta_{2 n+1} \rightarrow 0 \quad \text { as } \eta \rightarrow \infty,
\end{gathered}
$$

for some constants $\bar{\alpha}_{2 n}, \bar{\alpha}_{0}^{\prime}$. If equation (2.8c) is multiplied by $\alpha_{2 n}(\eta),(2.8 \mathrm{~d})$ by $\beta_{2 n+1}(\eta)$, the results added and summed over $n$, then one integration over $0 \leq \eta \leq \infty$ proves that $(2.8 \mathrm{c}, \mathrm{d})$ subject to $(2.13 \mathrm{a}, \mathrm{b})$ only admit a solution if $T_{1}$ satisfies the condition

$$
\begin{gathered}
T_{1}\left\{\sum_{n=-\infty}^{\infty} \int_{0}^{\infty}\left(e^{-(1+\mathrm{i}) \eta} v_{2 n-1}+e^{-(1-\mathrm{i}) \eta} v_{2 n+1}\right) \alpha_{2 n} d \eta\right\} \\
=4 \mathrm{i}\left(\sum_{n=-\infty}^{\infty} n \bar{\alpha}_{2 n} C_{2 n}\right)+2 \bar{\alpha}_{0}^{\prime} C_{0} .
\end{gathered}
$$

System (2.14) for the adjoint functions was solved using a procedure identical in spirit to that used for tackling $(2.8 \mathrm{a}, \mathrm{b})$. A consistency check on the numerical solutions involved and, on the derivation of the adjoint system, was provided by the condition that the eigenvalue for $T_{0}$ coming from the adjoint system (2.14) must be the same as that coming from the direct problem, equations $(2.8 \mathrm{a}, \mathrm{b})$ with boundary conditions (2.9a,b). Using terms up to $\beta_{7}$ gave values for $T_{0}$ from the adjoint problem which agreed to eight figures with the previously calculated values. Having determined the leading order eigenfunctions, the adjoint functions and the constants $C_{2 n}, \bar{\alpha}_{0}^{\prime}$ and $\bar{\alpha}_{2 n}$, elementary manipulation leads to the estimate $T_{1} \approx 41.19$.

Figure 2 shows the comparison between the asymptotic prediction (2.7c) for long wavelength neutrally stable vortices and the numerical values determined by a full solution of the governing stability equations (2.6). It is noteworthy that as $a \rightarrow 0$ so the discrepancy between the asymptotic and numerical values diminish but it is also evident that the two-term asymptotic series $T \approx 12.284 a^{-2}+41.19 a^{-1}+\ldots$ is significantly more accurate than the one-term prediction. Indeed, the agreement between the two-term asymptote and the numerical results is so good that for a wide range of long wavelengths the former may be used with a high degree of precision. 


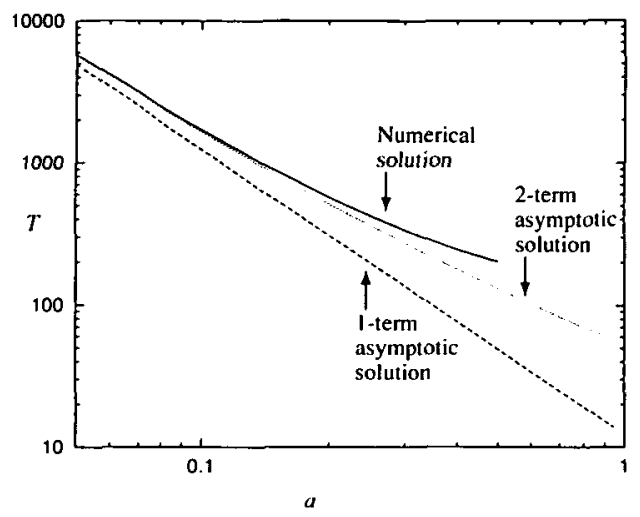

FIGURE 2. Comparison between numerically determined and asymptotic behaviour of the linear neutral stability curve for the Stokes layer on an oscillating cylinder. Plotted is the neutral Taylor number $T$ as a function of vortex wavenumber $a$. The solid line denotes this dependence according to a numerical solution of the full system (2.6) whilst the dashed and dotted lines illustrate the one and two-term asymptotic solutions as given by $(2.7 \mathrm{c})$.

\section{The sinusoidally heated flat plate problem}

In order to accentuate the analogy between this thermal flow and the curved Stokes flow described above we deduce the relevant equations for this second problem using a non-standard co-ordinate configuration. Consider a horizontal flat plate $\eta=0$ which is heated so that it has temperature $T_{\infty}+T_{L} \cos \omega t$. Suppose that above the plate lies an infinite fluid of thermal diffusivity $\kappa$ and kinematic viscosity $v$ and that lengths, velocities, pressure, time and temperature $T-T_{\infty}$ are made dimensionless by scaling by $\sqrt{2 \kappa / \omega}, \sqrt{2 \kappa \omega}, 2 \kappa \omega \rho_{\infty}, \omega$ and $T_{L}$ respectively. With the Prandtl number $\sigma$ given by $\sigma=\nu / \kappa$ and the Rayleigh number $R$ defined by

$$
R=\frac{\alpha g T_{L}}{2 \kappa \nu}\left(\frac{2 \kappa}{\omega}\right)^{\frac{3}{2}}
$$

where $\alpha$ is the coefficient of volume expansion and $g$ the gravitational constant, the basic conduction solution is given by $u=v=w=0, T=T_{B}=v_{B}$ where $v_{B}(\eta, t)$ is given by (2.3). If this basic flow is perturbed by solutions periodic in $z$ so that

$$
\begin{aligned}
& (u, v, w, p, T)=\left(0,0,0,0, T_{B}\right) \\
& \quad+\delta\{U(\eta, t) \cos a z, 0, W(\eta, t) \sin a z, P(\eta, t) \cos a z, \Theta(\eta, t) \cos a z\}
\end{aligned}
$$


then the disturbance equations can be reduced, under the standard Boussinesq approximation, to

$$
\begin{aligned}
\frac{\partial}{\partial t}\left(\frac{\partial^{2}}{\partial \eta^{2}}-a^{2}\right) U+\frac{1}{2} \sigma a^{2} R \Theta & =\frac{1}{2} \sigma\left(\frac{\partial^{2}}{\partial \eta^{2}}-a^{2}\right)^{2} U, \\
\frac{\partial \Theta}{\partial t}+U \frac{\partial T_{B}}{\partial \eta} & =\frac{1}{2}\left(\frac{\partial^{2}}{\partial \eta^{2}}-a^{2}\right) \Theta,
\end{aligned}
$$

subject to the boundary conditions $U=\Theta=\frac{\partial U}{\partial \eta}=0$ on $\eta=0$ and as $\eta \rightarrow \infty$.

Similarities clearly exist between the sets of equations (2.5) and (3.2) and the analysis of this thermal problem is started by imposing a Fourier decomposition of the velocity and temperature fields,

$$
(U, \Theta)=\sum_{n=-\infty}^{\infty}\left(U_{n}(\eta), \Theta_{n}(\eta)\right) e^{i n t} .
$$

With this form, (3.2) reduces to the infinite set of ordinary differential equations

$$
\begin{array}{r}
\left(D^{2}-a^{2}-\frac{2 \mathrm{i} n}{\sigma}\right)\left(D^{2}-a^{2}\right) U_{n}-a^{2} R \Theta_{n}=0, \\
\left(D^{2}-a^{2}-2 \mathrm{i} n\right) \Theta_{n}+(1+\mathrm{i}) e^{-\eta(1+\mathrm{i})} U_{n+1}+(1-\mathrm{i}) e^{-\eta(1-\mathrm{i})} U_{n-1}=0, \\
U_{n}=D U_{n}=\Theta_{n}=0 \text { on } \eta=0 \text { and } \text { as } \eta \rightarrow \infty .
\end{array}
$$

Hall [2] considered this system, in slightly different scalings, and calculated the critical conditions for linear stability. Blennerhassett $e t$ al. [1] attempted to extend these calculations to larger and smaller wavenumbers, but again encountered numerical difficulties. In this paper we consider only the resolution of the small wavenumber situation. As with the oscillating cylinder problem, the computational difficulties arise due to the slow decay of the velocity and temperature perturbations as the wave number tends to zero, and the emergence of a two layer structure to the perturbation fields. Before proceeding with the small wavenumber limit, we note that the system (3.3) exhibits the conjugation property that $U_{-n}=U_{n}^{*}, \Theta_{-n}=\Theta_{n}^{*}$, following from the requirement that $U$ and $\Theta$ are real functions, but the form of the terms coming from the interaction of the basic temperature field and the velocity perturbation prevents the occurrence of two decoupled solutions as in the oscillating cylinder case. Thus, in general, it must be expected that the solution of (3.2) contains all the harmonics of both $U$ and $\Theta$.

Following the procedure applied to the oscillating cylinder problem, the velocity and temperature perturbation fields are expanded as

$$
U_{n}=u_{n, 0}(\eta)+a u_{n, 1}(\eta)+\ldots, \quad \Theta_{n}=\theta_{n, 0}+a \theta_{n .1}(\eta)+\ldots,
$$


in the region where $\eta$ is $O(1)$, whilst the neutral Rayleigh number becomes

$$
R=a^{-2} R_{0}+a^{-1} R_{1}+\ldots
$$

The substitution of these expansions into (3.3) leads to the systems

$$
\begin{aligned}
\left(D^{2}-\frac{2 \mathrm{i} n}{\sigma}\right) D^{2} u_{n, 0}-R_{0} \theta_{n, 0} & =0, \\
\left(D^{2}-2 \mathrm{i} n\right) \theta_{n, 0}+(1+\mathrm{i}) e^{-\eta(1+\mathrm{i})} u_{(n-1), 0}+(1-\mathrm{i}) e^{-\eta(1-\mathrm{i})} u_{(n+1), 0} & =0,
\end{aligned}
$$

for the leading order terms, whilst the correction terms satisfy the equations

$$
\begin{aligned}
\left(D^{2}-\frac{2 \mathrm{i} n}{\sigma}\right) D^{2} u_{n, 1}-R_{0} \theta_{n, 1} & =R_{1} \theta_{n .0}, \\
\left(D^{2}-2 \mathrm{i} n\right) \theta_{n, 1}+(1+\mathrm{i}) e^{-\eta(1+\mathrm{i})} u_{(n-1), 1}+(1-\mathrm{i}) e^{-\eta(1-\mathrm{i})} u_{(n+1), 1} & =0 .
\end{aligned}
$$

The leading order problem $(3.5 \mathrm{a}, \mathrm{b})$ must be solved subject to conditions requiring the perturbations to vanish on the plate, viz

$$
u_{n, 0}=D u_{n, 0}=\theta_{n, 0}=0 \quad \text { at } \quad \eta=0,
$$

and matching conditions as $\eta$ tends to $\infty$. Here the equations $(3.5 \mathrm{a}, \mathrm{b})$ do not completely decouple as $\eta$ becomes large and, in the mean flow components, a linear growth with $\eta$ in $\theta_{0,0}$ and an $\eta^{4}$ behaviour for $u_{0,0}$ is allowed by the equations. For $n \neq 0$ the temperature must decay exponentially as $\eta$ tends to infinity, while, at most, $u_{n, 0}$ can grow linearly with $\eta$. Despite these possibilities, it was found that consistent matching between the wall and far field region could only be obtained if the perturbations satisfy the conditions

$$
\left(u_{0,0}-E_{0} \eta^{2}\right) / \eta^{2} \rightarrow 0, \quad u_{n, 0} \rightarrow E_{n} \quad(n \neq 0), \quad \theta_{0, n} \rightarrow 0 \quad \forall n \quad \text { as } \quad \eta \rightarrow \infty
$$

for some constants $E_{n}$. The quadratic growth in the mean component of the velocity field as $\eta \rightarrow \infty$ is arrested in the zone where $\eta=O\left(a^{-1}\right)$.

In this outer layer where $Y \equiv a \eta$ is $O(1)$, the solution has the form

$$
U_{0}=a^{-2} \hat{u}_{0,0}(Y)+\ldots, \quad U_{n}=\hat{u}_{n, 0}(Y)+\ldots(n \neq 0), \quad \Theta_{0}=a^{2} \hat{\theta}_{0,0}(Y)+\ldots,
$$

with $\Theta_{n}$ exponentially small for $n \neq 0$. Elementary manipulation reveals that in order to match with the large $\eta$ solutions (3.6b),

$$
\hat{u}_{0.0}=E_{0} Y^{2} e^{-Y}, \quad \hat{u}_{n .0}=E_{n} e^{-Y} \quad(n \neq 0), \quad \hat{\theta}_{0.0}=\frac{8 E_{0}}{R_{0}} e^{-Y} .
$$


These outer solutions now enable us to specify the requisite boundary conditions for the first order system $(3.5 \mathrm{c}, \mathrm{d})$. In addition to the usual vanishing of perturbations on the plate, i.e. $u_{n .1}=D u_{n .1}=\theta_{n .1}=0$ on $\eta=0$, it is necessary that

$$
\left(u_{0.1}+E_{0} \eta^{3}\right) / \eta^{2} \rightarrow \text { const., } \quad u_{n .1}+E_{n} \eta \rightarrow \text { const. } \quad(n \neq 0), \quad \theta_{n .1} \rightarrow 0 \quad \forall n,
$$

as $\eta \rightarrow \infty$.

The eigenproblem $(3.5 \mathrm{a}, \mathrm{b}, 3.6 \mathrm{a}, \mathrm{b})$ was solved numerically, on a finite domain $\left(0, \eta_{\infty}\right)$, using a similar AUTO routine to that implemented for the oscillating cylinder system $(2.8 \mathrm{a}, \mathrm{b})$. The growth in the mean flow component meant that the boundary conditions had to be imposed with some care. The condition $D^{3} u_{0.0}=0$ at $\eta_{\infty}$ allowed the required quadratic growth in $u_{0.0}$, but left no room to apply other conditions on $u_{0.0}$, despite the fact that $D^{4} u_{0.0}$ is present in the governing equations. The required two constraints at $\eta_{\infty}$ for the mean terms were provided by overspecifying the mean temperature $\theta_{0.0}$ : the conditions

$$
\theta_{0.0}=D \theta_{0.0}=0 \quad \text { at } \quad \eta=\eta_{\infty}
$$

were used in the calculations. For the higher harmonics the boundary conditions used at $\eta_{\infty}$ were asymptotic boundary conditions, derived using the projection techniques presented by Lentini and Keller [3]. Figure 3 shows the forms of the first few harmonics of the solution of this leading order eigenproblem for the choice of Prandtl number $\sigma=1$. It is noted that the sizes of the velocity harmonics are typically much greater than the corresponding temperature ones but that the mean velocity component is a dominant feature; for large $\eta$ it is found that $u_{0.0} \sim 81.53 \eta^{2}+O(\eta)$.

The leading order term in the Rayleigh number expansion was found to be $R_{0} \approx$ 209.91 , based on calculations including up to $\theta_{10.0}$ and $u_{10.0}$. It was also found that the convergence of $R_{0}$ as the number of terms in the Fourier series grows was much slower in this thermal problem than in the previous example.

The adjoint system to (3.5a,b), (3.6) may be cast as

$$
\begin{aligned}
\left(D^{2}-2 \mathrm{i} n\right) \gamma_{n}-R_{0} \delta_{n} & =0, \\
\left(D^{2}-\frac{2 \mathrm{i} n}{\sigma}\right) D^{2} \delta_{n}+(1+\mathrm{i}) e^{-\eta(1+\mathrm{i})} \gamma_{n+1}+(1-i) e^{-\eta(1-\mathrm{i})} \gamma_{n-1} & =0,
\end{aligned}
$$

subject to $\gamma_{n}=\delta_{n}=D \delta_{n}=0$ on $\eta=0$ for all $n$ together with $\delta_{n} \rightarrow \bar{\delta}_{n}$ as $\eta \rightarrow \infty$ for some constants $\bar{\delta}_{n}$. It should be noted that these far-field conditions force $\gamma_{n} \rightarrow$ constant for $n \neq 0$ (solutions with exponential growth must be excluded) and $\gamma_{0}$ may grow quadratically for large $\eta$. If we follow the usual procedure of multiplying (3.5c) by $\gamma_{n}(\eta),(3.5 \mathrm{~d})$ by $\delta_{n}(\eta)$, adding the resulting equations and then summing over $n$, we 

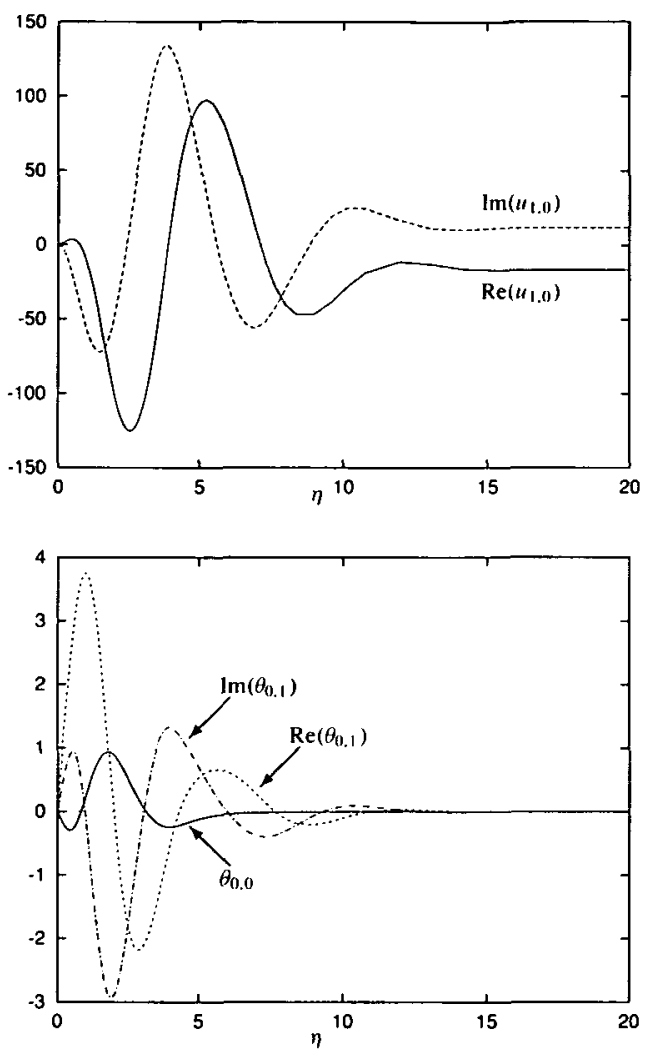

FIGURE 3. The first few components of the eigenfunctions for the leading order thermal problem (3.5a,b), (3.6); a) The real (solid line) and imaginary (dashed) parts of the fundamental $u_{1.0}(\eta)$; b) The mean temperature $\theta_{0.0}$ (solid) and real (dashed) and imaginary (chain) parts of $\theta_{0.1}$.

deduce, on application of the boundary conditions (3.9), that (3.5c,d) admit a suitable solution only if the Rayleigh number correction term satisfies the equation

$$
\sigma R_{\mathbf{I}}\left\{\sum_{n=-\infty}^{\infty} \int_{0}^{\infty} \theta_{n, 0}(\eta) \delta_{n}(\eta) d \eta\right\}=2 \mathrm{i}\left(\sum_{n=-\infty}^{\infty} n \bar{\delta}_{n} E_{n}\right) .
$$

Solution of the adjoint system (3.10) together with numerical evaluation of the integrals and constants in (3.11) yields the value $R_{1}=3.77$. Figure 4 shows a comparison between the two term asymptotic form of the neutral Rayleigh number $R \approx 209.91 a^{-2}+3.77 a^{-1}$ and numerical values determined by solution of the full governing system (3.2). As in the previous example, it is noted that the asymptotic 
result is increasingly useful as $a \rightarrow 0$ and that this approximation is good over an appreciable range of long vortex wavelengths.

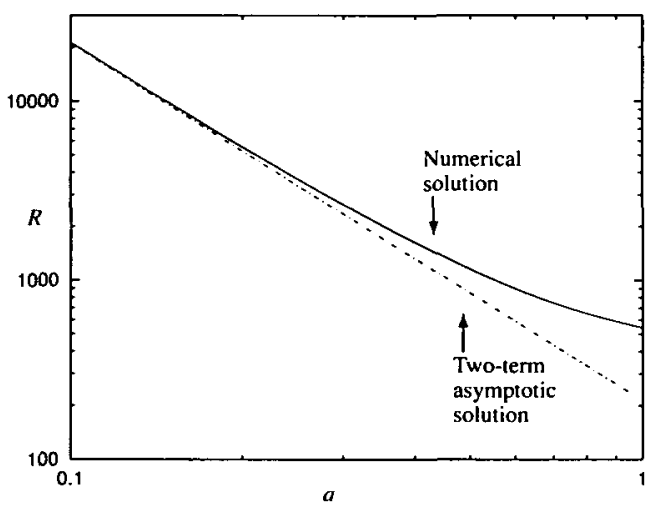

FIGURE 4. Comparison between the numerically determined (solid line) and the two-term small-a asymptote $R \approx 209.91 a^{-2}+3.77 a^{-1}$ to the linear neutral stability curve for the sinusoidally heated plane layer.

\section{Discussion}

In this work we have endeavoured to explore the linear neutral stability properties of vortex modes in two particular time-periodic flows. It has been shown that expansions in the (assumed small) vortex wavenumber, combined with the method of adjoints, yields the first two terms in the expression for the neutral curve. A complicating factor is that in both cases examined the solution structure divides into two distinct regimes with the slow exponential decay of the disturbances taking place in the outer of these. It is this feature which is the principal reason that makes direct numerical solution of the governing linear stability equations difficult using the Fourier series decomposition procedure. For small wavenumbers the domain over which the full equations must be integrated in order to capture the correct far-field decay is very large.

The two problems we have chosen to look at share a number of important common features but, nevertheless, illustrate a few important differences. The thermal problem is harder to solve than the centripetal one. This is partly because the leading order system does not decouple into two smaller ones and partly because the quadratic growth in the mean velocity field (see (3.6b)) is more difficult to impose numerically than the corresponding linear growth required by the centripetal problem. Our solutions show 
that the two-term small $a$ asymptotes provide good agreement with numerical results over large parameter ranges; in the centripetal problem the two-term asymptote is significantly more accurate that the one-term result. In contrast, for the thermal case, the two-term asymptote is graphically indistinguishable from the one-term result, a feature which can be attributed to the observation that since $R_{0} \gg R_{1}$, the second term $R_{1} a^{-1}$ in the small $a$ expansion for the neutral Rayleigh number is swamped by the leading order one. We have seen that in both cases the mean components of the disturbance functions are huge compared with the harmonics. However, in the centripetal case the sizes of the scaled radial and azimuthal velocity components are broadly similar (see Figure 1) whilst for the heated problem the induced velocity components overwhelm both the mean and fluctuating temperature disturbances.

To conclude, we have successfully described the stability properties of long wavelength vortices in two related oscillatory flows. Our results suggest that it is relatively straightforward to obtain useful asymptotic expressions for the behaviour of the neutral curve and our technique, combined with the Fourier decomposition numerical approach for $a=O(1)$ and the large wavenumber studies of [1], enables us to discuss rigorously the linear stability of vortex modes across the complete spectrum of wavenumbers. Moreover, the basis laid here should be easily extendible in order to describe the stability characteristics of vortices in a large class of time-periodic flows.

\section{Acknowledgements}

This work was undertaken whilst A.P.B. was visiting the School of Mathematics, University of New South Wales. He is indebted to the Royal Society of London and the Australian Research Council without whose grants his visit would not have been possible. In addition, he is grateful both to the staff and students of New College and to the School of Mathematics, UNSW for their hospitality.

We thank the referees for their comments relating to the presentation of this work.

\section{References}

[1] P. J. Blennerhassett, J. P. Denier and A. P. Bassom, "High wavenumber vortices in time-periodic flows", submitted to Proc. R. Soc. Lond. A.

[2] P. Hall, "Instability of time-periodic flows", in: Stability of time dependent and spatially varying flows (eds. D. L. Dwoyer and M. Y. Hussaini), (Springer, Berlin, 1987) 206-224.

[3] M. Lentini and H. B. Keller, "Boundary value problems on semi-infinite intervals and their numerical solution", SIAM J. Numer. Anal 17 (1980) 577-604.

[4] G. Seminara and P. Hall, "Centrifugal instability of a Stokes layer: linear theory", Proc. R. Soc. Lond. A 350 (1976) 299-316. 